\title{
One-Bit Precoding and Constellation Range Design for Massive MIMO with QAM Signaling
}

\author{
Foad Sohrabi, Student Member, IEEE, Ya-Feng Liu, Member, IEEE and Wei Yu, Fellow, IEEE
}

\begin{abstract}
The use of low-resolution digital-to-analog converters (DACs) for transmit precoding provides crucial energy efficiency advantage for massive multiple-input multiple-output (MIMO) implementation. This paper formulates a quadrature amplitude modulation (QAM) constellation range and one-bit symbol-level precoding design problem for minimizing the average symbol error rate (SER) in downlink massive MIMO transmission. A tight upper-bound for SER with low-resolution DAC precoding is first derived. The derived expression suggests that the performance degradation of one-bit precoding can be interpreted as a decrease in the effective minimum distance of the QAM constellation. Using the obtained SER expression, we propose a QAM constellation range design for the singleuser case. It is shown that in the massive MIMO limit, a reasonable choice for constellation range with one-bit precoding is that of the infinite-resolution precoding with per-symbol power constraint, but reduced by a factor of $\sqrt{2 / \pi}$ or about 0.8 . The corresponding minimum distance reduction translates to about $2 \mathrm{~dB}$ gap between the performance of one-bit precoding and infinite-resolution precoding. This paper further proposes a low-complexity heuristic algorithm for one-bit precoder design. Finally, the proposed QAM constellation range and precoder design are generalized to the multi-user downlink. We propose to scale the constellation range for infinite-resolution zero-forcing (ZF) precoding with per-symbol power constraint by the same factor of $\sqrt{2 / \pi}$ for one-bit precoding. The proposed one-bit precoding scheme is shown to be within $2 \mathrm{~dB}$ of infinite-resolution ZF. In term of number of antennas, one-bit precoding requires about $50 \%$ more antennas to achieve the same performance as infinite-resolution precoding.
\end{abstract}

\section{INTRODUCTION}

Massive multiple-input multiple-output (MIMO) systems in which the base station (BS) is equipped with large-scale antenna arrays, e.g., in the order of several hundreds of antennas, is a promising candidate for the next generation wireless systems for achieving high spectral efficiency, reliability, and connectivity requirements [1]. One of the main challenges in designing the downlink massive MIMO transmission scheme is that the implementation of the conventional digital precoding strategies such as [2]-[5] may not be practical,

F. Sohrabi, and W. Yu are with The Edward S. Rogers Sr. Department of Electrical and Computer Engineering, University of Toronto, Toronto, ON M5S 3G4, Canada (e-mail: fsohrabi@comm.utoronto.ca; weiyu@comm.utoronto.ca). Y.-F. Liu is with the State Key Laboratory of Scientific and Engineering Computing, Institute of Computational Mathematics and Scientific/Engineering Computing, Academy of Mathematics and Systems Science, Chinese Academy of Sciences, Beijing 100190, China (email: yafliu@1sec.cc.ac.cn). This paper has been presented in part at the Information Theory and Applications (ITA) Workshop, San Diego, CA, U.S.A February 2018. This work is supported in part by the Natural Science and Engineering Research Council (NSERC) of Canada, a Steacie Fellowship, in part by Qualcomm Technologies, Inc. and in part by the National Natural Science Foundation of China under Grants 11671419 and 11688101. because conventional precoding schemes require one dedicated high-resolution digital-to-analog converters (DACs) for each antenna element. When a BS transmitter is equipped with large number of antennas, this leads to high hardware complexity and excessive circuit power consumption.

To address the above issue, one line of works [6]-[9] propose hybrid precoding architecture in which the precoder consists of two parts: low-dimensional digital precoder and high-dimensional analog precoder. In the hybrid precoding, the number of required DACs scales with the number of data streams rather than the number of antennas [8]. Although the hybrid precoding scheme has been shown to be capable of approaching the performance of the conventional digital zero-forcing (ZF) precoding scheme [9], hybrid precoding still requires high-resolution DACs. As the power consumption of DAC grows exponentially with the resolution [10], [11], the use of high-resolution DAC can lead to high power consumption, especially when the BS needs to transmit a large number of data streams.

This paper considers an alternative precoding architecture called one-bit precoding in which two DACs (i.e., one DAC for converting the in-phase signal and the other one for converting the quadrature signal) are dedicated for each antenna element but with only one-bit resolution. The one-bit precoding enables us to reduce the circuit power consumption of the DACs. Moreover, the one-bit precoding satisfies the condition of constant envelop transmission, hence it can prevent the possible amplitude distortions which may occur in the practical systems when the power amplifiers work in the saturation region [12].

\section{A. Main Contributions}

This paper considers the precoder design problem for the downlink of a multi-user multiple-input single-output (MISO) system where only one-bit resolution DACs are available at the transmitter. In particular, we recognize that due to the one-bit DAC, precoding needs to be done on a symbol-bysymbol basis. Moreover, we observe that it is crucial to design the quadrature amplitude modulation (QAM) constellation range for each given channel realization in order to perform low-resolution transmit precoding for each symbol of the designed QAM constellation with reasonable accuracy. This paper focuses on the massive MIMO regime and uses the average symbol error rate (SER) of uncoded transmission at the receiver as the design criterion. We make the following main technical contributions toward the goal of optimizing the constellation range and subsequently one-bit symbol-level precoding for the massive MIMO downlink: 
Tight SER Expression: A tight expression for the SER for QAM transmission under one-bit symbol-level precoding is derived. Due to the low-resolution transmit DAC, the noiseless receive signal may not be exactly at the intended constellation location. The derived expression suggests that there is a reduction in the minimum constellation symbol distance in this case. The minimum distance is effectively reduced by the twice of the distance between the noiseless received signal and the intended constellation symbol.

QAM Constellation Range Design: We point out the importance of optimizing the QAM constellation range with one-bit symbol-level precoding. Inspired by the results from the case where the infinite-resolution DACs are available, we conclude that the constellation range should be restricted to the regime where the noiseless received signal of one-bit precoding can approach all the points in the designed constellation. Our analytical result shows that a reasonable choice for the QAM constellation range for one-bit precoding should be about $\sqrt{2 / \pi}$ times the optimal constellation range in the infiniteresolution case with instantaneous (i.e., per-symbol) power constraint in the massive MIMO limit.

Low-Complexity Algorithm for Precoding Design: In one-bit precoding, the transmitting signal of each antenna is selected from a quadrature phase shift keying (QPSK) alphabet and hence the transmitting signal design problem is a combinatorial optimization problem for which exhaustive search would have exponential complexity. This paper proposes a low-complexity two-step heuristic algorithm which finds a high-quality solution for the one-bit symbol-level precoding problem. In the first step, the algorithm iteratively designs the transmitted signal at each antenna such that the overall residual signal is as close to the origin of the complex plane as possible. This process is repeated until the transmit signals of all the antennas except last few, i.e., about $5-10$ antennas, are designed. In the second step, the algorithm performs an exhaustive search on the transmit signals of the remaining antennas in order to find the best match to the intended constellation point. The complexity of the proposed algorithm scales with the square of the total number of antennas.

Multi-User One-Bit Precoding: The proposed constellation range and one-bit precoder designs are generalized to the multi-user case. We first design the constellation range for the multi-user zero-forcing precoder with per-symbol power constraint but assuming infinite-resolution DACs. We observe that in the limit of large number of users, the constellation range for the multi-user case should be set to be that of the single-user case multiplied by a factor which is a function of the number of constellation points and the number of users. For the one-bit precoding case, we propose to further scale the proposed constellation range design by $\sqrt{2 / \pi}$, the same factor as for the single-user massive MIMO. The lowcomplexity precoding design algorithm is likewise generalized to the multi-user scenario.

Performance Characterization: We analytically characterize the performance of the proposed one-bit symbol-level precoding design and show that in the massive MIMO regime there is about $2 \mathrm{~dB}$ gap between the proposed one-bit precoding scheme and infinite-resolution precoding (under the same per- symbol power constraint). This $2 \mathrm{~dB}$ gap is true for both singleuser and multi-user precoding designs. Moreover, we show that this $2 \mathrm{~dB}$ gap translates to requiring about $50 \%$ more antennas for the one-bit precoding architecture as compared to the conventional infinite-resolution architecture for achieving the same performance.

\section{B. Related Work}

Most of the existing literatures on one-bit beamforming focuses on the uplink scenario in which the BS is equipped with one-bit analog-to-digital converters (ADCs). The performance analyses of such systems for narrowband channels are provided in [13] $-[16]$ while those for wideband channels are presented in [17], [18].

The early works on downlink precoding with one-bit DACs propose to use linear-quantized precoding schemes in which the precoder is obtained by quantizing the traditional linear precoders [19]-[21]. Although the class of linear-quantized precoding can approach the performance of the infiniteresolution precoding in the low signal-to-noise ratio (SNR) regime, it suffers from a high symbol error floor in the high SNR regime. In [22], the authors propose some methods for slightly perturbing the transmitted signal of the quantized $\mathrm{ZF}$ scheme in [20], [21] in order to improve performance at higher SNRs and numerically show that those perturbations can provide significant gains for QPSK signaling. More sophisticated non-linear precoding methods have been proposed in $[23]-[26]$ for the scenario where both the receivers and the transmitter are equipped with one-bit ADCs and DACs, respectively. However, these methods are applicable only for QPSK signaling and for the case where the receiver has onebit ADC. We observe that in practice the user equipment is often equipped with relatively few number of antennas, so the use of high-resolution ADCs at the downlink receiver is often acceptable from a power consumption point of view. For this reason, this paper considers the scenario with one-bit DAC at the transmitter, but high-resolution $\mathrm{ADC}$ at the receiver.

The authors of [27] generalize the algorithm in [23] for the infinite-resolution receivers and introduce a technique to transmit 16-QAM symbols with one-bit transmitters based on the idea of superposition coding. Unlike the algorithm in [27], the proposed scheme in this paper can be applied to the QAM constellations with any size. More importantly, this paper points out the crucial role played by QAM constellation range design, which is not considered in prior work [27].

The recent contributions of [28], [29] consider designing the constellation range, as well as a non-linear precoder scheme with higher order modulations. The algorithms in [28], [29], which seek to minimize the mean squared error at the user side, achieve excellent performance within few $\mathrm{dB}$ gap to the infinite-resolution precoding benchmarks. However, in the algorithm of [28], [29], the constellation range is designed for each symbol transmission, which means that the range information needs to be communicated to the receiver as side information on a symbol-by-symbol basis. This is a significant overhead. The recent work [30] extends the algorithm in [29] by considering the constellation range design for $T$ symbol 
transmissions in which the channel remains constant. However, the high computational complexity of the algorithm in [30] (which is $O\left(M^{3} T^{3}\right)$ for a system with $M$ transmit antennas) prohibits its use for the typical massive MIMO systems in which $M$ and $T$ are both large.

By recognizing that the coherence time of the wireless channel in practice is typically large enough to allow at least several hundreds of symbol transmissions, this paper proposes to design the constellation range for each fading block such that it is suitable for all possible symbol vector choices from the constellation. Furthermore, this paper shows that for largescale antenna arrays the proposed constellation range design can be approximated by a constant value which is independent of the channel realization thanks to the "channel hardening" phenomenon in massive MIMO systems [31]. Therefore, the QAM constellation range can be predetermined a priori with no need for instantaneous channel state information (CSI) at the receivers.

It is worth mentioning that the factor $\sqrt{2 / \pi}$, which we propose as a constellation range reduction factor for onebit precoding as compared to the infinite-resolution precoding scenario, also appears in the results of [20], [29], but for a different scheme of linear-quantized precoding, and using a completely different justification.

Finally, one-bit precoding for signal constellations other than QAM constellation has also been considered, e.g., [32], [33] propose novel one-bit precoding designs for the phase shift keying (PSK) constellation. Comparing the performance of different constellation choices and finding the best constellation can be considered as an interesting direction for future work.

\section{Paper Organization and Notations}

The remainder of this paper is organized as follows. Section III introduces the system model and the problem formulation for massive MIMO one-bit precoding. Section III is devoted to deriving the SER expression in one-bit precoding architecture. For the single-user case, Section IV considers designing the QAM constellation range while Section V considers designing the precoder for the fixed constellation range. Those designs are further generalized to the multi-user case in Section VI, where the performance gap between the proposed one-bit precoding design and the infinite-resolution $\mathrm{ZF}$ precoder is also analyzed. Simulation results are provided in Section VII and conclusions are drawn in Section VIII

This paper uses lower-case letters for scalars, lower-case bold face letters for vectors and upper-case bold face letters for matrices. The real part of a complex scalar $s$ is denoted by $\operatorname{Re}\{s\}$. Further, we use the superscript ${ }^{H}$ to denote the Hermitian transpose of a matrix and superscript $\dagger$ to denote the complex conjugate. The identity and all-one matrices with appropriate dimensions are denoted by $\mathbf{I}$ and $\mathbf{1}$, respectively; $\mathbb{C}^{m \times n}$ denotes an $m$ by $n$ dimensional complex space; $\mathcal{C N}(\mathbf{0}, \mathbf{R})$ represents the zero-mean circularly symmetric complex Gaussian distribution with covariance matrix $\mathbf{R}$; $\mathcal{N}(\boldsymbol{\mu}, \boldsymbol{\Sigma})$ represents a real Gaussian distribution with mean $\boldsymbol{\mu}$ and covariance matrix $\Sigma$. The notations $\operatorname{Tr}(\cdot), \log _{10}(\cdot)$, and

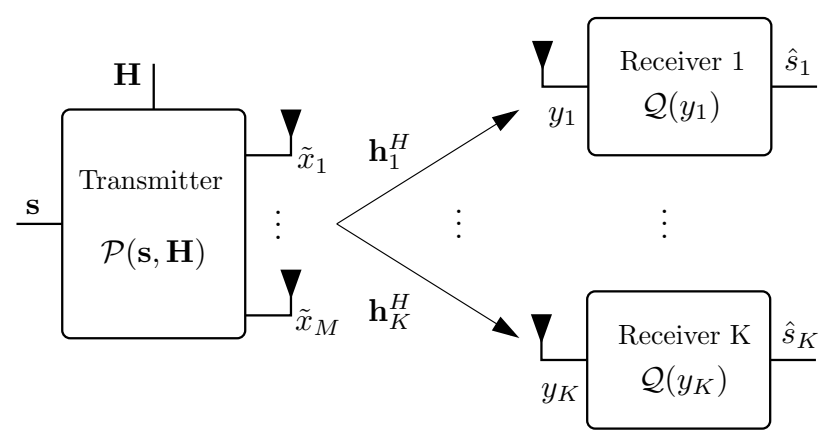

Fig. 1: A multi-user MISO system with symbol-level precoding.

$\mathbb{E}\{\cdot\}$ represent the trace, decimal logarithm, and expectation operators, respectively. Finally, $|\cdot|$ represents the absolute value of a scaler while $\|\cdot\|_{p}$ indicates the $p$-norm of a vector.

\section{SySTEM MOdEL}

Consider the downlink of a multi-user MISO system in which a BS with a large number of antennas, $M$, serves $K$ single-antenna users. For such a system, the received signal at user $k$ can be modeled as

$$
y_{k}=\sqrt{\frac{P}{M}} \mathbf{h}_{k}^{H} \mathbf{x}+z_{k},
$$

where $\mathbf{h}_{k}^{H} \in \mathbb{C}^{1 \times M}$ is the vector of channel gains to user $k, \mathbf{x} \in \mathbb{C}^{M \times 1}$ is the normalized transmitted signal, $z_{k} \sim$ $\mathcal{C N}\left(0,2 \sigma^{2}\right)$ is the additive white Gaussian noise, and $P$ is the total transmit power budget.

This paper seeks to design the transmitted signal when onebit DACs are employed at each antenna element. This means that the normalized transmitted signal $\mathrm{x}$ must come from a finite alphabet, i.e., $\mathbf{x} \in \mathcal{X}^{M}$, where $\mathcal{X}=\left\{\frac{1}{\sqrt{2}}( \pm 1 \pm \imath)\right\}$, where $\imath$ is the imaginary unit satisfying $\imath^{2}=-1$. In order to focus on the impact of one-bit precoding, this paper assumes that the full CSI is available at the transmitter.

In conventional infinite-resolution precoding, the transmitted signal is designed as a product of a beamforming vector and the symbol constellation point. This is not possible to do when the transmitted signal must come from a lowresolution alphabet. This paper considers instead a symbollevel precoding scheme, in which the BS designs the $M$ dimensional precoded transmitted signal, $\tilde{\mathbf{x}} \triangleq \sqrt{\frac{P}{M}} \mathbf{x}$, directly on a symbol-by-symbol basis as a function of the instantaneous channel state information (CSI), $\mathbf{H}=\left[\mathbf{h}_{1}, \ldots, \mathbf{h}_{K}\right]^{H}$, and the intended constellation point, $\mathbf{s}=\left[s_{1}, \ldots, s_{K}\right]$, as

$$
\tilde{\mathbf{x}}=\mathcal{P}(\mathbf{s}, \mathbf{H}),
$$

where the function $\mathcal{P}: \mathbb{C}^{M} \times \mathbb{C}^{K \times M} \rightarrow \mathcal{X}^{M}$ represents the precoder.

At the receiver side, we do not assume the availability of the CSI, and instead assume that in each coherence block of the channel, the constellation range and the constellation size are fed back to the users such that each user seeks to recover its intended symbol, $s_{k}$, from its received signal, $y_{k}$, by mapping $y_{k}$ to its nearest constellation point, i.e., $\hat{s}_{k}=\mathcal{Q}\left(y_{k}\right)$. The overall system model is shown in Fig. 1. Note that the 
concept of symbol-level precoding is considered in the earlier works [34]-[40] for the infinite-resolution case-it is adopted for the finite-resolution DAC context considered in this paper. We note here that symbol-level precoding requires transmit beamforming adaptation at the symbol rate, rather than at the timescale of channel coherence time as in the case of traditional beamforming. This translates to significantly more demanding transmit processing speed requirement.

This paper restricts attention to uncoded square QAM constellations and also explicitly designs the constellation range for one-bit symbol-level precoding. In particular, we use $N^{2}$ QAM constellations with the range of $c$ in each dimension and the minimum distance of $d=\frac{c}{N-1}$; an example for $N=4$ is depicted in Fig. 2. We assume a block-fading channel model in which the channel stays constant for at least hundreds of symbol transmissions so that the constellation range $c$ and the constellation size $N$ only need to be communicated to the receiver as preamble on a per-fading-block basis. It is emphasized that this paper considers the adaptation of the constellation range to the channel state information only, while the number of constellation points are assumed to be fixed. A complete adaptive modulation scheme in which both the constellation range and the number of constellation points are jointly optimized can be considered as future work. We also emphasize that the techniques presented in this paper pertain to uncoded transmission only. A complete characterization of the capacity and the optimal transmission strategy for the finite-resolution-input massive MIMO channel is still an open problem.

In general, for the multi-user scenario, different constellations can be designed for different users. However, similar to the references [28]-[30], this paper considers a simplified problem in which the symbols of all users come from the same constellation. This assumption may force the users with strong channels to operate below their actual capacities, but such fairness concern can be addressed by an intelligent scheduler that groups users with nearly similar channels within each time-frequency slot. For this reason, the rest of the paper assumes that all users have the same large-scale fading, i.e., $\mathbf{h}_{k} \sim \mathcal{C N}(\mathbf{0}, \mathbf{I}), \forall k$.

The problem of interest is to design the QAM constellation range in each coherence time of the channel, and then design the transmitted signal corresponding to each symbol vector where each element of that symbol vector is chosen from the designed constellation in order to minimize the average symbol error rate. The proposed constellation range design for the multi-user scenario is based on the i.i.d Rayleigh fading channel model in which a similar large-scale fading is assumed for all the users. Generalizing this design for other channel models require further efforts and it can be considered as future work.

\section{SER ChARACTERIZATION FOR SYMBOL-LEVEL PRECODING}

This section provides an SER expression of a generic user in a multi-user MISO system with one-bit symbol-level precoding described in Section III. In order to avoid encumbering the

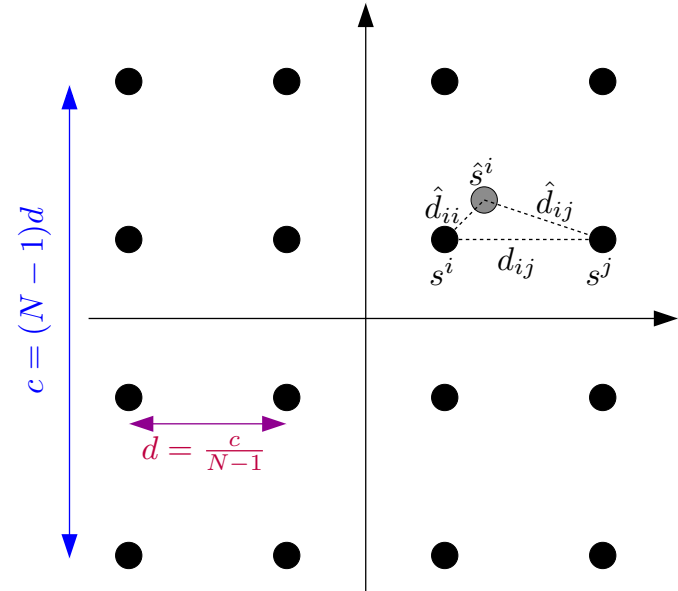

Fig. 2: An example of $N^{2}$-QAM constellation for $N=4$.

notation, we drop the user index, $k$, in the notations throughout this section.

Let us assume that the intended constellation point for the considered receiver is $s^{i}$. Due to the finite-resolution DAC constraint in one-bit precoding, the noiseless received signal, $\hat{s}^{i}=\sqrt{\frac{P}{M}} \mathbf{h}^{H} \mathbf{x}_{i}$, may not exactly coincide with the intended constellation point. In this case, the received signal is $y=$ $\hat{s}^{i}+z$ and the pairwise error probability, $\operatorname{Pr}\left(s^{i} \rightarrow s^{j}\right)$, can be written as

$$
\begin{aligned}
\operatorname{Pr}\left(s^{i} \rightarrow s^{j}\right) & \triangleq \\
& \stackrel{(a)}{=} \operatorname{Pr}\left(\left|y-s^{i}\right| \geq\left|y-s^{j}\right|\right) \\
& \stackrel{(b)}{=} \operatorname{Pr}\left(\operatorname{Re}\{\tilde{z}\} \geq \frac{\left|\hat{s}^{i}-s^{j}\right|^{2}-\left|\hat{s}^{i}-s^{i}\right|^{2}}{2\left|s^{i}-s^{j}\right|}\right) \\
& \stackrel{(c)}{=} \quad Q\left(\frac{\hat{d}_{i j}^{2}-\hat{d}_{i i}^{2}}{2 d_{i j} \sigma}\right),
\end{aligned}
$$

where $Q(u)=\frac{1}{\sqrt{2 \pi}} \int_{u}^{\infty} e^{-\frac{v^{2}}{2}} d v, \tilde{z}=z e^{\imath \angle\left(s^{j}-s^{i}\right)^{\dagger}}$ is a Gaussian random variable with the same distribution as $z$, i.e., $\tilde{z} \sim \mathcal{C N}\left(0,2 \sigma^{2}\right), \hat{d}_{i j} \triangleq\left|\hat{s}^{i}-s^{j}\right|$, and $d_{i j} \triangleq\left|s^{i}-s^{j}\right|$. In the above equations, $(a)$ is obtained by substituting $y=\hat{s}^{i}+z$, (b) is obtained by defining $\tilde{z}=z e^{\imath \angle\left(s^{j}-s^{i}\right)^{\dagger}}$ and rearranging the inequality for $\tilde{z}$, and $(c)$ is based on the definition of the $Q$-function.

Similar to the conventional SER analysis for a reasonable SNR range [41], the pairwise probability error of the closest neighboring constellation points in (3) can be used to tightly approximate the overall SER as

$$
\mathrm{SER} \approx \frac{1}{N^{2}} \sum_{i} \sum_{j \in \overline{\mathcal{N}}_{i}} Q\left(\frac{\hat{d}_{i j}^{2}-\hat{d}_{i i}^{2}}{2 d_{i j} \sigma}\right),
$$

where $\overline{\mathcal{N}}_{i}$ is the set of nearest constellation points to $s^{i}$.

The expression in (4) is complicated and is difficult to use as a metric to design the constellation range. As a result, we seek to find an upper-bound for the expression in (4). As it can be seen from Fig. 2, $\hat{d}_{i i}, \hat{d}_{i j}$, and $d_{i j}$ can be considered 
as three edges of a triangle, and hence by using the triangular inequality, we can write

$$
\begin{aligned}
\frac{\hat{d}_{i j}^{2}-\hat{d}_{i i}^{2}}{2 d_{i j}} & =\frac{\left(\hat{d}_{i j}+\hat{d}_{i i}\right)\left(\hat{d}_{i j}-\hat{d}_{i i}\right)}{2 d_{i j}} \\
& \geq \frac{\left(d_{i j}\right)\left(d_{i j}-2 \hat{d}_{i i}\right)}{2 d_{i j}}=\frac{d_{i j}-2 \hat{d}_{i i}}{2} .
\end{aligned}
$$

Now, using that $Q(\cdot)$ is a decreasing function and $d_{i j}=d$ for all $j \in \overline{\mathcal{N}}_{i}$ where $d$ denotes the minimum distance in the considered QAM constellation, we can write the following upper-bound on the SER expression in (4) as:

$$
\mathrm{SER} \lesssim \sum_{i} \frac{g_{i}}{N^{2}} Q\left(\frac{d-2 \hat{d}_{i i}}{2 \sigma}\right)
$$

where $g_{i}$ is the number of minimum-distance neighbors of the symbol $s^{i}$.

The rest of the paper first considers designing the constellation range and the non-linear precoder for the single-user scenario, $K=1$, by minimizing the expression (6), then generalizes the proposed design to the multi-user case.

\section{Constellation Range Design for Single-User SCENARIO}

This section proposes an appropriate choice of constellation range, $c$, for one-bit symbol-level precoding under a fixed MISO channel serving a single user. In order to gain insight on how to design $c$ for one-bit precoding, we first consider designing the constellation range of symbol-level precoding with infinite-resolution DACs under instantaneous per-symbol total power constraint across the antennas, and also under instantaneous per-symbol per-antenna power constraint. The instantaneous per-symbol power constraint refers to that each precoded symbol needs to satisfy a power constraint (instead of the average power across multiple symbols.)

\section{A. Infinite-Resolution Precoding with Total Power Constraint}

Under symbol-level precoding, the transmit signal is designed as a function of the constellation point and the instantaneous channel realization. The range of the QAM constellation is an important parameter that needs to be designed to optimize performance. Assuming a square $N^{2}$-QAM and using the expression (6) as the metric for minimizing the SER with $d=\frac{c}{N-1}$ and $\hat{d}_{i i}=\left|\sqrt{\frac{P}{M}} \mathbf{h}^{H} \mathbf{x}_{i}-s^{i}\right|$, the problem of optimizing the constellation range for symbol-level precoding with infinite-resolution precoding and with instantaneous persymbol total power constraint can be written as

$$
\min _{c \geq 0} \sum_{i=1}^{N^{2}} \frac{g_{i}}{N^{2}} \min _{\left\|\mathbf{x}_{i}\right\|_{2}^{2} \leq M} Q\left(\frac{\frac{c}{N-1}-2\left|\sqrt{\frac{P}{M}} \mathbf{h}^{H} \mathbf{x}_{i}-s^{i}\right|}{2 \sigma}\right) .
$$

With infinite resolution on $\mathbf{x}_{i}$, the optimal solution $\mathbf{x}_{i}$ for the inner minimization problem of (7) with fixed $c$ is

$$
\mathbf{x}_{i}=\frac{\sqrt{M} \mathbf{h}}{\|\mathbf{h}\|_{2}} \min \left\{1, \frac{\left|s^{i}\right|}{\sqrt{P}\|\mathbf{h}\|_{2}}\right\} e^{\imath \angle s^{i}}
$$

Intuitively, the optimal $\mathbf{x}_{i}$ is to match the channel. With infinite resolution on $\mathbf{x}_{i}$, the precoded signal can reconstruct $s^{i}$ perfectly as long as

$$
\left|s^{i}\right| \leq \sqrt{P}\|\mathbf{h}\|_{2} .
$$

By substituting this solution for all $\mathbf{x}_{i}$ in (7) and taking some simple algebraic steps, the problem (7) for designing $c$ can be rewritten as

$\min _{c \geq 0} \sum_{i} \frac{g_{i}}{N^{2}} Q\left(\frac{\frac{c}{N-1}-2 \max \left\{0,\left|s^{i}\right|-\sqrt{P}\|\mathbf{h}\|_{2}\right\}}{2 \sigma}\right)$.

In 10 , the Q-function can be approximated by $Q(x) \approx$ $\frac{1}{12} e^{-\frac{x^{2}}{2}}+\frac{1}{4} e^{-\frac{2}{3} x^{2}}, x>0[42]$. This exponential relation between the output of the $Q$-function and its argument suggests that the value of the objective function in 10 is dominated by the error probability of symbols which result in the smallest argument of the $Q$-function. Further, since $Q(\cdot)$ is a decreasing function, the smallest argument occurs for the furthest constellation points which have the maximum $\left|s^{i}\right|$, i.e., $\max _{i}\left|s^{i}\right|=\frac{c}{\sqrt{2}}$. Therefore, it is desirable to consider designing the constellation range such that the argument of the $Q$-function for those furthest constellation points is maximized, i.e.,

$$
\max _{c \geq 0}\left\{\frac{c}{N-1}-2 \max \left\{0, \frac{c}{\sqrt{2}}-\sqrt{P}\|\mathbf{h}\|_{2}\right\}\right\} .
$$

The objective function of (11) is a piecewise linear function of $c$ and the optimal solution for that, $c_{\mathrm{inf}, \mathrm{t}}^{*}$, can be calculated as

$$
c_{\mathrm{inf}, \mathrm{t}}^{*}=\sqrt{2 P}\|\mathbf{h}\|_{2} .
$$

This result has the following interpretation. As mentioned earlier, with infinite-resolution symbol-level precoding under instantaneous total power constraint, we can construct any point in the complex plane inside a circle centered at the origin of the complex plane with radius $\sqrt{P}\|\mathbf{h}\|_{2}$ exactly as the noiseless received signal. The $\sqrt{2}$ factor comes from the fact that the distance of the furthest constellation point from the origin for a square constellation is $\frac{c}{\sqrt{2}}$. The expression in 12 suggests that we should increase the range of the constellation, $c$, as much as possible subject to the constraint that the furthest constellation point can be constructed by symbol-level precoding. In other words, the furthest constellation points should be designed to be at the distance of $\sqrt{P}\|\mathbf{h}\|_{2}$ from the origin.

The constellation range in 12 is a function of the channel realization, $\mathbf{h}$. This means that $c_{\mathrm{inf}, \mathrm{t}}^{*}$ should adapt to the realization of the channel in each coherence time. However, for large-scale antenna arrays, it is possible to show that $\|\mathbf{h}\|_{2}$ can be well approximated by a constant $\sqrt{M}$. This phenomenon, called channel hardening in massive MIMO literature [31], suggests that for large $M$ we can approximate

$$
c_{\mathrm{inf}, \mathrm{t}}^{*} \stackrel{M}{\approx} \underset{ }{2} \sqrt{2 P M}
$$

without significant performance degradation. By this design, the constellation range is a function of the path-loss only and not specific Rayleigh fading component of $\mathbf{h}$. 


\section{B. Infinite-Resolution Precoding with Per-Antenna Power Constraint}

This paper eventually considers a one-bit precoder for which every antenna has the same transmit power, so as an intermediate step, it is worth considering infinite-resolution precoding with per-antenna power constraint rather than total power constraint. In this case, the normalized transmitted signal of each antenna should satisfy $\left|x_{m}\right| \leq 1$.

Fixing $\mathbf{h}$, we claim that the complex numbers that can be realized with $\sqrt{\frac{P}{M}} \mathbf{h}^{H} \mathbf{x}$ under the constraints $\left|x_{m}\right| \leq 1, \forall m$, are exactly all the points inside a circle centered at the origin of the complex plane, but with a reduced radius as compared to the total power constraint case. To show this, let us consider the following optimization problem:

$$
\min _{\left|x_{m}\right| \leq 1, \forall m}\left|\sqrt{\frac{P}{M}} \mathbf{h}^{H} \mathbf{x}-s\right| .
$$

The optimal solution for $\mathbf{x}$ in 14 is given as

$$
x_{m}=\min \left\{\sqrt{\frac{M}{P\|\mathbf{h}\|_{1}}}|s|, 1\right\} e^{\imath\left(\angle h_{m}+\angle s\right)}, \quad \forall m,
$$

where $h_{m}$ is the $m^{\text {th }}$ element of $\mathbf{h}$. Now by substituting (15) into the objective function of (14), we can see that only for complex numbers $s$ inside a circle of $|s| \leq \sqrt{\frac{P}{M}}\|\mathbf{h}\|_{1}$, it is possible to realize $s$ exactly. We denote the radius of this circle by

$$
r^{*}=\sqrt{\frac{P}{M}}\|\mathbf{h}\|_{1}
$$

We remark that $r^{*}$ is also the largest range of $\sqrt{\frac{P}{M}} \mathbf{h}^{H} \mathbf{x}$, i.e.,

$$
r^{*}=\max _{\left|x_{m}\right| \leq 1, \forall m}\left|\sqrt{\frac{P}{M}} \mathbf{h}^{H} \mathbf{x}\right| .
$$

This interpretation will be useful in the constellation range design for one-bit precoding.

Using a similar argument as in Section IV-A it can be shown that the optimal constellation range for minimizing the SER is the largest constellation range such that all the constellation points can be constructed accurately. Therefore, the furthest constellation point, which is at the distance $\frac{c}{\sqrt{2}}$ for a square constellation, should be at a distance $r^{*}$ from the origin, yielding that the optimal constellation range is

$$
c_{\mathrm{inf}, \mathrm{p}}^{*}=\sqrt{\frac{2 P}{M}}\|\mathbf{h}\|_{1} .
$$

For the considered channel model in which each element of the channel vector has an i.i.d Gaussian distribution, i.e., $h_{j} \sim \mathcal{C} \mathcal{N}(0,1)$, we have $\mathbb{E}\left\{\left|h_{j}\right|\right\}=\sqrt{\pi / 4}$. Now, using the law of large number in the large-scale antenna array limit when $M \rightarrow \infty$, we can write $\frac{\|\mathbf{h}\|_{1}}{M} \rightarrow \sqrt{\pi / 4}$. Therefore, in systems with massive antenna arrays, it is possible to approximate $\frac{\|\mathbf{h}\|_{1}}{\sqrt{M}}$ as $\sqrt{(\pi / 4) M}$. This result can be used to further simplify the constellation range expression in 18 as

$$
c_{\mathrm{inf}, \mathrm{p}}^{*} \stackrel{M}{\approx} \approx \infty \sqrt{\pi / 4} \sqrt{2 P M}
$$

It can be seen from (13) and (19) that the ratio between the constellation ranges in infinite-resolution precoding under total power constraint and under per-antenna power constraint in the massive MIMO limit converges to a constant:

$$
\frac{c_{\text {inf,p }}^{*}}{c_{\text {inf,t }}^{*}} \rightarrow \sqrt{\pi / 4}, \quad \text { as } \quad M \rightarrow \infty,
$$

i.e., under the per-antenna power constraint, the constellation size should be reduced by about $88 \%$ in order for the symbollevel precoder to be able to synthesize the constellation point.

\section{One-Bit Precoding}

We now consider one-bit symbol-level precoding where $\mathbf{x} \in \mathcal{X}^{M}$ in which $\mathcal{X}=\left\{\frac{1}{\sqrt{2}}( \pm 1 \pm \imath)\right\}$. Unlike the infiniteresolution case, the realizations of $\sqrt{\frac{P}{M}} \mathbf{h}^{H} \mathbf{x}$ is no longer a continuum. Thus, it is no longer always true that all constellation points can be constructed exactly at the transmitter. Nevertheless, as long as the reconstruction error is sufficiently small (e.g., below the noise level), one-bit precoding can still provide good performance.

To gain some intuition, Fig. 3 is a scatter plot of all $4^{M}$ possible realizations of $\mathbf{h}^{H} \mathbf{x}$ for the case of $M=8$ antennas for a fixed $\mathbf{h}$. It can be seen that all these points are confined in a circle centered at the origin, yet the radius of the circle in which these points concentrate is further reduced as compared to the infinite-resolution cases discussed earlier.

This paper aims to make a case that similar to the infiniteresolution case, i.e., (17), if the constellation range is designed such that the furthest constellation point is located at $\bar{r}^{*}$, where

$$
\bar{r}^{*}=\max _{\mathbf{x} \in \mathcal{X}^{M}}\left|\sqrt{\frac{P}{M}} \mathbf{h}^{H} \mathbf{x}\right|,
$$

then all the constellation points can be reconstructed accurately. This is clearly a necessary condition for constellation range. But, as shown numerically later in the paper, when the number of transmit antennas $M$ is large, this is also a sufficient condition, i.e., an appropriate one-bit precoding design can approach any complex number inside the circle with radius $\bar{r}^{*}$ with negligible error.

Following this design strategy, the constellation range design problem for one-bit precoding can be written as

$$
c_{1 \text {-bit }}^{*}=\sqrt{2} \max _{\mathbf{x} \in \mathcal{X}^{M}}\left|\sqrt{\frac{P}{M}} \mathbf{h}^{H} \mathbf{x}\right| .
$$

The numerical evaluation of the above maximization is, unfortunately, difficult. Instead of solving this problem directly, this paper proposes to reduce the constellation range for one-bit precoding (which automatically satisfies per-antenna power constraint) as compared to infinite-resolution precoding with per-antenna power constraint by a constant factor. The following result [43] helps us identify such a factor.

Consider the following optimization problem

$$
\begin{array}{ll}
\max _{\mathbf{x}} & \mathbf{x}^{H} \mathbf{A x} \\
\text { s.t. } & x_{m} \in \mathcal{F}_{q}, \quad \forall m,
\end{array}
$$




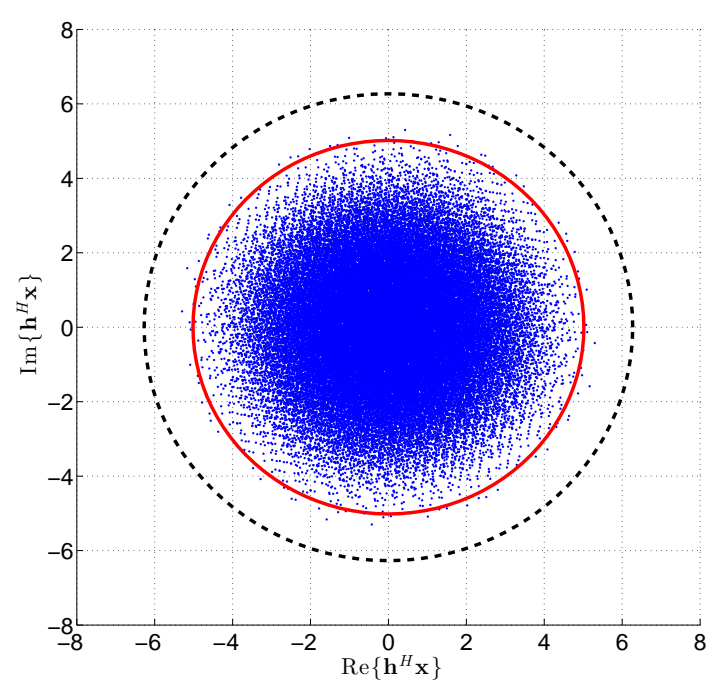

Fig. 3: Fix one random channel realization with $M=8$ and $P=4$. The data points are the $4^{M}$ possible realizations of $\mathbf{h}^{H} \mathbf{x}$, and the dashed black and solid red lines are circles with radius of $c_{\text {inf,t }}^{*}=\sqrt{2 P}\|\mathbf{h}\|_{2}$ and $\sqrt{2 / \pi} c_{\text {inf, }}^{*}$, respectively. Most data points are confined within the solid red circle.

where $\mathcal{F}_{q}=\left\{1, e^{\imath \frac{2 \pi}{q}}, \ldots, e^{\imath \frac{2 \pi(q-1)}{q}}\right\}$. Further, consider the following semidefinite programming (SDP) relaxation of 23] which is now a convex optimization problem,

$$
\begin{array}{cl}
\max _{\mathbf{X} \in \mathbb{C}^{M \times M}} & \operatorname{Tr}(\mathbf{A X}) \\
\text { s.t. } & \mathbf{X} \succeq \mathbf{0}, \\
& \mathbf{X}(i, i)=1, \quad \forall i=1, \ldots, M .
\end{array}
$$

Suppose $\mathbf{X}^{*}$ is an optimal solution of problem (24). To generate a feasible $\mathrm{x}$ from the solution of problem (24), draw a random vector $\zeta \sim \mathcal{C N}\left(\mathbf{0}, \mathbf{X}^{*}\right)$, and then quantize each element of $\boldsymbol{\zeta}$ to the nearest point in $\mathcal{F}_{q}$. The expectation of the objective function of the solution obtained using the above randomization procedure can be shown to be greater than $\alpha_{q} \operatorname{Tr}\left(\mathbf{A X}^{*}\right)$ [43], where

$$
\alpha_{q}= \begin{cases}\frac{2}{\pi}, & \text { if } q=2, \\ \frac{q^{2}\left(1-\cos \frac{2 \pi}{q}\right)}{8 \pi}, & \text { if } q \geq 3 .\end{cases}
$$

For the given $q$, let us denote the objective function in 23 for the optimal solution as $f_{q}^{*}$ when $\mathbf{A}=\frac{P}{M} \mathbf{h} \mathbf{h}^{H}$. Then, the constellation range for infinite-resolution precoding with perantenna power constraint and one-bit precoding can be written as $c_{\text {inf,p }}^{*}=\sqrt{2 f_{\infty}^{*}}$ and $c_{1-\text { bit }}^{*}=\sqrt{2 f_{4}^{*}}$, respectively. Now since it is difficult to exactly characterize $f_{4}^{*}$, we propose to approximate $\frac{f_{4}^{*}}{f_{\infty}^{*}}$ by the ratio of the SDP approximation bounds $\frac{\alpha_{4}}{\alpha_{\infty}}=\frac{2 / \pi}{\pi / 4}=8 / \pi^{2}$. This means that

$$
c_{1-\mathrm{bit}}^{*} \approx \sqrt{8 / \pi^{2}} c_{\mathrm{inf}, \mathrm{p}}^{*} \approx \sqrt{2 / \pi} c_{\mathrm{inf}, \mathrm{t}}^{*},
$$

where the last approximate equality follows from 20 .

To see that the proposed constellation range is an appropriate choice, let us consider as an example of a relatively small system with $M=8$ antennas and $P=4$. For a randomly

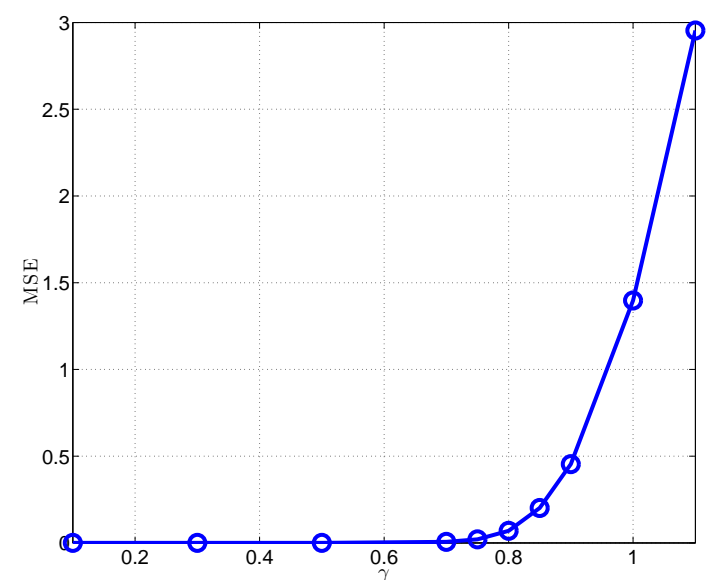

Fig. 4: The MSE versus $\gamma$ for a system with $M=8$ and $P=4$. There is a phase transition at $\gamma_{\mathrm{th}}=\sqrt{2 / \pi} \approx 0.8$.

generated fixed h, Fig. 3 plots all $4^{M}$ possible realizations of $\mathbf{h}^{H} \mathbf{x}$. Fig. 3 also plots two circles: a dashed black circle with radius of $c_{\mathrm{inf}, \mathrm{t}}^{*}=\sqrt{2 P}\|\mathbf{h}\|_{2}$ and a solid red circle with the radius of $\sqrt{2 / \pi} c_{\mathrm{inf}, \mathrm{t}}^{*}$ where the latter is the proposed value for $c_{1-\text { bit }}^{*}$. It can be seen visually that almost all the realizations of $\mathbf{h}^{H} \mathbf{x}$ are located inside the solid red circle suggesting that the proposed design is a necessary condition for constellation range.

To see that the proposed design is also a sufficient condition for constellation range, let us define the mean squared error (MSE) as

$$
\operatorname{MSE}(s)=\mathbb{E}_{\mathbf{h}}\left\{\left|\sqrt{\frac{P}{M}} \mathbf{h}^{H} \mathbf{x}-s\right|^{2}\right\},
$$

and the parameter

$$
\gamma=\frac{|s|}{\sqrt{P}\|\mathbf{h}\|_{2}} .
$$

Fig. 4 plots the average MSE against the parameter $\gamma$ over $10^{3}$ channel realizations. The precoder $\mathrm{x} \in \mathcal{X}^{M}$ is found by exhaustive search in order to minimize the squared error for given $s$ and $\mathbf{h}$. It can be seen from Fig. 4 that there is a phase transition around $\gamma_{\text {th }}=\sqrt{2 / \pi} \approx 0.8$ before which the MSE is very small, while the MSE starts to increase significantly after $\gamma_{\text {th }}$. This means that any complex number inside the circle with radius $c_{1-\text { bit }}^{*}=\sqrt{2 / \pi} \sqrt{2 P}\|\mathbf{h}\|_{2}$ can be realized accurately, while it is infeasible to do so for complex numbers outside of that circle, thereby justifying the choice $c_{1 \text {-bit }}^{*}=\sqrt{2 / \pi} c_{\text {inf,t }}^{*}$. Further numerical evidence is provided in Section VII to support this choice under practical precoding and when $M$ is large.

\section{One-Bit Precoding Design for Single-User SCENARIO}

This section proposes a practical algorithm for designing the single-user one-bit symbol-level precoder, or equivalently the normalized transmitting signal, $\mathbf{x}_{i}$, corresponding to each symbol, $s^{i}$, assuming fixed constellation range $c$. Using (6) as the SER metric, it can be seen that the only term that 
depends on the precoder for a fixed constellation is $\hat{d}_{i i}=$ $\left|\sqrt{\frac{P}{M}} \mathbf{h}^{H} \mathbf{x}_{i}-s^{i}\right|$. Since the $Q$-function is a decreasing function, the transmit signal design problem for the $i^{\text {th }}$ symbol can be written as

$$
\mathbf{x}_{i}^{*}=\underset{\mathbf{x}_{i} \in \mathcal{X}^{M}}{\operatorname{argmin}}\left|\sqrt{\frac{P}{M}} \mathbf{h}^{H} \mathbf{x}_{i}-s^{i}\right| .
$$

Solving this optimization problem is hard due to the combinatorial nature of its constraints. Here, we seek to find a good solution for 29) with low complexity.

Toward this aim, we observe that for a fixed channel $\mathbf{h}$, the possible realizations of $\mathbf{h}^{H} \mathbf{x}$, when $\mathbf{x} \in \mathcal{X}^{M}$, are densely distributed close to the origin; an example for $M=8$ is depicted in Fig. 3. This observation suggests that if we can choose the transmitting signal across a suitably chosen subset of antennas in a greedy fashion so as to bring the residual to be small, then we can use exhaustive search at the remaining (e.g. 8) antennas to drive the residual very close to zero. Based on this, we propose the following two-step algorithm to find a reasonable solution for (29):

- Step 1: Use an iterative greedy approach to bring the residual close to zero by designing the precoded signal at a suitably chosen subset of $M_{1}=M-M_{2}$ antennas where $M_{1} \gg M_{2}$. In particular, at each iteration, the algorithm selects one antenna and its corresponding transmitting signal to minimize the norm of the residual. This procedure is executed until the transmitting signals for $M_{1}$ antennas are all determined.

- Step 2: Design the transmitting signals of the other $M_{2}$ antennas to further approximate the desired signal by performing an exhaustive search. Note that the exhaustive search is feasible as $M_{2} \ll M$.

The mathematical details of the above two-step algorithm are illustrated in Algorithm 1. The overall computational complexity of the algorithm is $O\left(M M_{1}\right)+O\left(4^{M_{2}}\right)$, where the first and the second terms correspond to the first and the second steps of the proposed algorithm, respectively. In our numerical results, we observe that $5 \leq M_{2} \leq 10$ is a reasonable range of choices for $M_{2}$ which provides good performance with reasonable computational complexity.

\section{Vi. Constellation Range and One-Bit Precoding DESIGN FOR MUlTI-USER SCENARIO}

This section generalizes the proposed designs for the QAM constellation range and the transmitting signals in Section IV and Section $\mathrm{V}$ from the single-user scenario to the multi-user scenario.

\section{A. Constellation Range Design}

When a multi-antenna BS serves multiple users at the same time, the constellation range depends not only on the channel, but also on the number of users $K$ being served simultaneously and the constellation size. This section provides an analysis of the optimal constellation range in the massive MIMO regime under multi-user symbol-level precoding. We begin by considering the infinite-resolution $\mathrm{ZF}$ scheme with per-symbol total power constraint across the antennas.

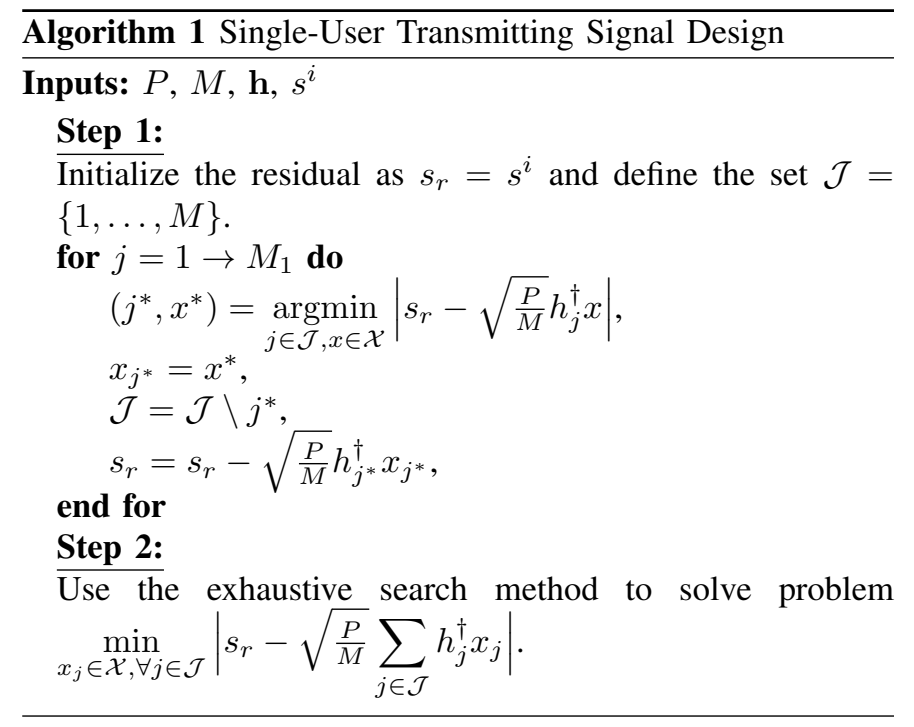

Fixing a channel realization $\mathbf{H}$, the $\mathrm{ZF}$ precoding vector can be found by solving the following optimization problem which illustrates the minimum required power so that the noiseless received signal of each user is exactly equal to its intended signal, namely,

$$
\begin{array}{ll}
\min _{\mathbf{x}} & \|\mathbf{x}\|_{2}^{2} \\
\text { s.t. } & \sqrt{\frac{P}{M}} \mathbf{H} \mathbf{x}=\mathbf{s} .
\end{array}
$$

This problem is convex and its optimal solution is given by

$$
\mathbf{x}^{*}=\sqrt{\frac{M}{P}} \mathbf{H}^{H}\left(\mathbf{H} \mathbf{H}^{H}\right)^{-1} \mathbf{s} .
$$

The constellation range should be designed such that for a vector of intended constellation points, $\mathbf{s}=\left[s_{1}, \ldots, s_{K}\right]$, the normalized transmitted signal satisfies $\left\|\mathbf{x}^{*}\right\|_{2}^{2} \leq M$. This is equivalent to

$$
\mathbf{s}^{H}\left(\mathbf{H H}^{H}\right)^{-1} \mathbf{s} \leq P .
$$

Now using the pseudo-orthogonal property of the channels of different users in the large-scale massive MIMO regime, we can approximate $\left(\mathbf{H H}^{H}\right)^{-1}$ with a diagonal matrix in which the $k^{\text {th }}$ diagonal element is $\frac{1}{\left\|\mathbf{h}_{k}\right\|_{2}^{2}}$. By approximating $\left(\mathbf{H} \mathbf{H}^{H}\right)^{-1}$ with that diagonal matrix, the inequality (32) can be rewritten as

$$
\sum_{k=1}^{K} \frac{\left|s_{k}\right|^{2}}{\left\|\mathbf{h}_{k}\right\|_{2}^{2}} \leq P
$$

This constraint can be further simplified by taking advantage of the channel hardening phenomenon in the massive MIMO regime that allows the approximation $\left\|\mathbf{h}_{k}\right\|_{2}^{2} \approx M$ for all $k$ as

$$
\sum_{k=1}^{K}\left|s_{k}\right|^{2} \leq P M
$$

The constellation range design problem is then to choose the range $c$ so that the above constraint is satisfied for most realizations of $\left(s_{1}, s_{2}, \ldots, s_{K}\right)$ if they are within the range.

Consider a particular user $k$ whose intended signal $s_{k}$ is selected in an independent and identically distributed (i.i.d.) 
fashion from an $N^{2}$-QAM constellation with range $c$. A routine calculation shows that

$$
\mu_{s}=\mathbb{E}\left\{\left|s_{k}\right|^{2}\right\}=\frac{N+1}{6(N-1)} c^{2},
$$

and

$$
\sigma_{s}^{2}=\mathbb{E}\left\{\left(\left|s_{k}\right|^{2}-\mu_{s}\right)^{2}\right\}=\frac{(N+1)\left(N^{2}-4\right)}{90(N-1)^{3}} c^{4} .
$$

When a large number of users are precoded together, by the central limit theorem, we have that

$$
\sqrt{K}\left(\frac{1}{K} \sum_{k=1}^{K}\left|s_{k}\right|^{2}-\mu_{s}\right) \rightarrow \mathcal{N}\left(0, \sigma_{s}^{2}\right) .
$$

The optimal constellation range for $\mathrm{ZF}$ precoding is the maximum range $c$ such that the precoded signal almost always satisfies the per-symbol power constraint, i.e., 34. In this paper, we propose to design $c$ so that the mean of $\sum_{k=1}^{K}\left|s_{k}\right|^{2}$ in (34) is within two standard deviation from the constraint $P M$. This ensures that (34) is satisfied with high probability. Using (37) and the properties of the Gaussian distribution, this design goal of $K \mu_{s}+2 \sqrt{K} \sigma_{s}=P M$ yields that

$$
c_{\mathrm{ZF}}^{*}=\sqrt{\frac{2 P M}{f(K, N)}},
$$

where

$$
f(K, N)=K \frac{N+1}{3(N-1)}+2 \sqrt{K \frac{(N+1)\left(N^{2}-4\right)}{22.5(N-1)^{3}}} .
$$

Comparing the constellation range design of the infiniteresolution $\mathrm{ZF}$ for the multi-user scenario with that of the single-user case considered in Section IV] i.e., (38) vs (13), it can be seen that there is an extra scaling factor $\sqrt{\frac{1}{f(K, N)}}$ in the constellation range expression when a massive MIMO BS simultaneously serves large number of users.

Finally, we consider the constellation range design for the one-bit precoding case. Inspired by the form of (38), this paper proposes to multiply the same scaling factor $\sqrt{\frac{1}{f(K, N)}}$ to our proposed single-user constellation range design for one-bit precoding in 26) to account for the effect of serving multiple users, i.e.,

$$
c_{1 \text {-bit }}^{*}=\sqrt{2 / \pi} \sqrt{\frac{2 P M}{f(K, N)}},
$$

Numerical results are presented later in the paper to show that this is an appropriate design.

\section{B. One-Bit Precoding Design}

We now present a practical algorithm for one-bit symbollevel precoder design for the multi-user MISO system. In the multi-user scenario where the intended symbol of each user is selected from a square $N^{2}$-QAM constellation, the vector of intended symbols has $N^{2 K}$ choices. For each choice of $\mathbf{s}^{i}$ where $i \in\left\{1, \ldots, N^{2 K}\right\}$, we seek to design the normalized

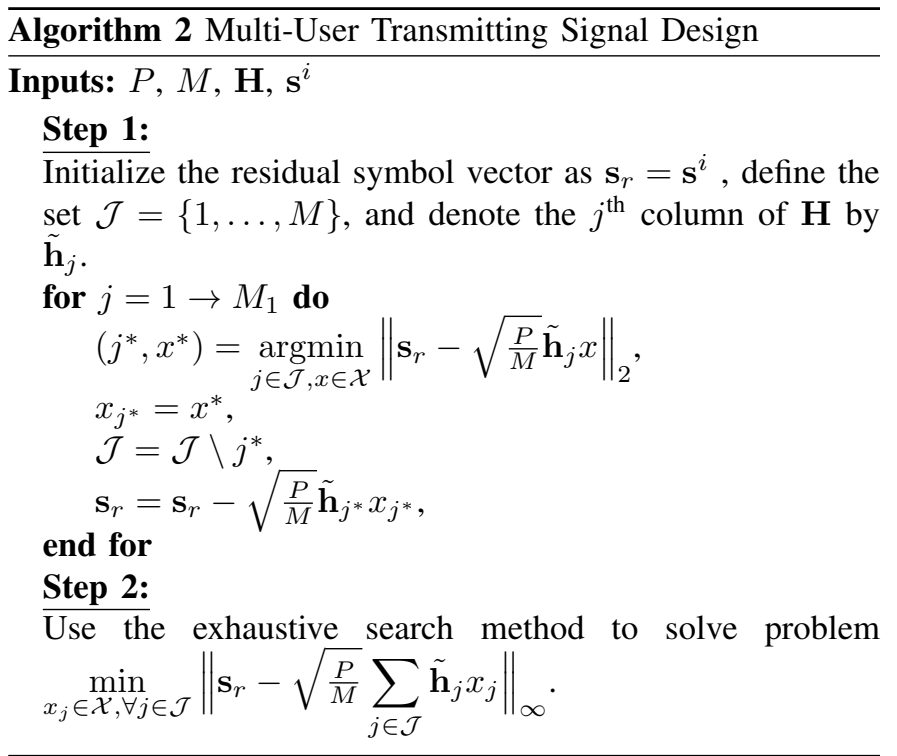

transmitting signal $\mathbf{x}_{i}$ to minimize the average user SER, mathematically,

$$
\min _{\mathbf{x}_{i} \in \mathcal{X}^{M}} \sum_{k} \frac{g_{k}^{i}}{N^{2}} Q\left(\frac{d_{1-\mathrm{bit}}-2\left|\sqrt{\frac{P}{M}} \mathbf{h}_{k}^{H} \mathbf{x}_{i}-s_{k}^{i}\right|}{2 \sigma}\right),
$$

where $d_{1 \text {-bit }}=\frac{c_{1 \text {-bit }}^{*}}{N-1}$ and $s_{k}^{i}$ is the $k^{\text {th }}$ element of $\mathbf{s}^{i}$. Instead of tackling the problem in (41), due to the rapidly decreasing shape of the $Q$-function, we can consider the following optimization problem

$$
\min _{\mathbf{x}_{i} \in \mathcal{X}^{M}}\left\|\sqrt{\frac{P}{M}} \mathbf{H} \mathbf{x}_{i}-\mathbf{s}^{i}\right\|_{\infty},
$$

which minimizes the maximum approximation error across all the users. For solving this problem, we use a generalization of the two-step algorithm proposed in Section $\mathrm{V}$ for the singleuser case.

The proposed two-step approach is summarized in Algorithm 2 Specifically, the first step of the proposed algorithm seeks to bring the residual vector close to the origin, i.e., minimize the 2-norm of the residual symbol vector, by choosing the transmit values over a subset of $M_{1}$ antennas in a greedy fashion. In the second step of the algorithm, exhaustive search is performed over the remaining $M_{2}=M-M_{1}$ antennas to minimize the maximum deviation from the residual symbols, i.e., to minimize the $\infty$-norm of the residual symbol vector. The rational behind minimizing the 2-norm in the first step is that the realizations of $\sqrt{\frac{P}{M}} \mathbf{H} \mathbf{x}$ for $\mathbf{x} \in \mathcal{X}^{M}$ are distributed densely close to the origin which means that the vectors with smaller 2-norm are surrounded by more realizations. Therefore, it is expected that the second step of the algorithm achieves a better performance if the residual symbol vector that is passed to it as an input has a smaller 2-norm.

The computational complexity of the first and the second steps of Algorithm 2 are $O\left(K M M_{1}\right)$ and $O\left(K 4^{M_{2}}\right)$, respectively. Simulation results presented in Section VII show excellent numerical performance of the algorithm with reasonable complexity. 


\section{Performance Gap of One-Bit Precoding vs. Infinite- Resolution ZF}

For infinite-resolution ZF under the instantaneous power constraint with the constellation range designed as 38 , since almost surely the constellation points can be reconstructed exactly, the symbol error rate simply scales with the minimum distance as follows:

$$
\mathrm{SER}=\bar{g}_{N} Q\left(\frac{d}{2 \sigma}\right),
$$

where $\bar{g}_{N}=4\left(1-\frac{1}{N}\right)$ is the average number of nearest neighbors in the symbol constellation, and $d$ is the minimum distance in the constellation, which can be expressed as:

$$
d_{\mathrm{ZF}}^{*}=\frac{c_{\mathrm{ZF}}^{*}}{N-1}=\sqrt{\frac{2 P M}{\tilde{f}(K, N)}},
$$

where $\tilde{f}(K, N)=(N-1)^{2} f(K, N)$.

In the one-bit precoding scheme, there is no guarantee that the noiseless received signals can exactly realize the intended symbols. However, if we design the constellation range carefully so that the one-bit precoder can approximate the noiseless receive signals to be very close to the intended signals, then the term $2 \hat{d}_{i i}$ in (6) is negligible as compared to the minimum distance $d$, and we can tightly approximate the SER of the proposed scheme using the same equation as 437 with

$$
d_{1-\text { bit }}=\frac{c_{1 \text {-bit }}^{*}}{N-1}=\sqrt{2 / \pi} \sqrt{\frac{2 P M}{\tilde{f}(K, N)}} .
$$

This suggests that the one-bit precoding scheme requires about $10 \log _{10} \frac{1}{2 / \pi} \approx 2 \mathrm{~dB}$ more power than the conventional $\mathrm{ZF}$ to achieve the same performance in the massive MIMO regime. The simulation results of this paper indeed show this $2 \mathrm{~dB}$ gap under the proposed constellation range design, thereby verifying that the proposed design is a reasonable one.

We can also characterize the performance gap of one-bit precoding and conventional $\mathrm{ZF}$ in terms of the number of extra antennas that would need to be added to one-bit precoding in order to achieve the same performance as conventional ZF. Using (43), it can be seen that

$$
M_{1-\mathrm{bit}}=\frac{1}{2 / \pi} M_{\mathrm{ZF}} \approx 1.57 M_{\mathrm{ZF}} .
$$

This means that one-bit precoding with about $50 \%$ more number of antennas can achieve the same performance as infinite-resolution ZF precoding under the same instantaneous per-symbol power constraint.

It is worth mentioning that although the above discussion pertains to the multi-user case, since the same constellation range design, i.e., $c_{1-\mathrm{bit}}^{*}=\sqrt{2 / \pi} c_{\mathrm{inf,t}}^{*}$ is used for the singleuser case, the performance gap between the infinite-resolution precoding with per-symbol total power constraint and the proposed one-bit precoding design for the single-user case is also about $2 \mathrm{~dB}$, which again translates to requiring about $50 \%$ more antennas for one-bit precoding to achieve the same performance as infinite-resolution precoding.

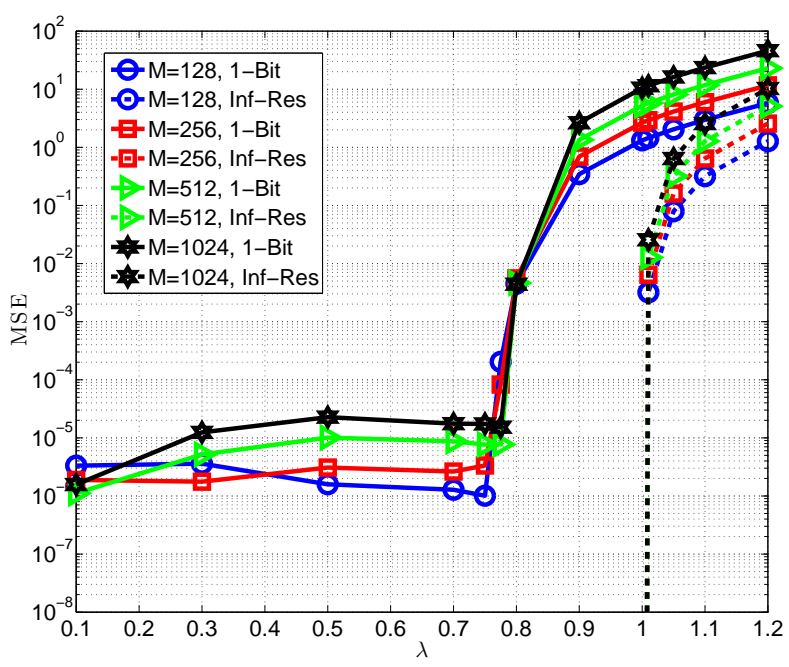

Fig. 5: Average MSE versus $\lambda$ in a single-user system for different numbers of antennas, $M$, when $P=1$ and $M_{2}=8$.

\section{Simulation Results}

In this section, we present numerical simulation results to support the proposed design methodology for constellation range and to evaluate the performance of the proposed algorithm for one-bit symbol-level precoding for both single-user and multi-user scenarios.

\section{A. MSE Analysis for Constellation Range Design}

First, we show that the constellation range design proposed in Section IV-C for the single-user case, which sets $c_{1 \text {-bit }}^{*}=$ $\sqrt{2 / \pi} \sqrt{2 P}\|\mathbf{h}\|_{2}$ is nearly optimal. Consider a BS with $M$ antennas serving one user by transmitting symbols from a 16QAM constellation with constellation range of $c$. We evaluate the average MSE defined in (27) against the parameter

$$
\lambda=\frac{c}{\sqrt{2 P}\|\mathbf{h}\|_{2}},
$$

where the average is over the channel realizations and onebit precoding is performed using Algorithm 11. The transmit power is set to $P=1$. The number of antennas $M$ ranges from 128 to 1024 . Note that $M_{2}$ is set to 8 in all cases in order to keep the complexity manageable.

Fig. 5 shows that there is a sharp phase transition in MSE for both infinite-resolution and one-bit precoding. The phase transition occurs at $\lambda=1$ for infinite-resolution precoding with instantaneous per-symbol total power constraint, thus verifying the constellation range design (12), i.e., $c_{\mathrm{inf}, \mathrm{t}}^{*}=\sqrt{2 P}\|\mathbf{h}\|_{2}$. For one-bit precoding, the phase transition occurs at about $\lambda=\sqrt{2 / \pi} \approx 0.8$, thus verifying the constellation range design 26 , i.e., $c_{1-\text { bit }}^{*}=\sqrt{2 / \pi} \sqrt{2 P}\|\mathbf{h}\|_{2}$ even with the proposed low-complexity practical one-bit precoding algorithm.

In Fig. 5 the MSE for the values of $\lambda$ smaller than the phase transition point indicates the quality of the one-bit precoder design. One may ask whether the MSE achieved by the proposed one-bit precoding is sufficiently small so that it does not significantly degrade the SER performance. The 


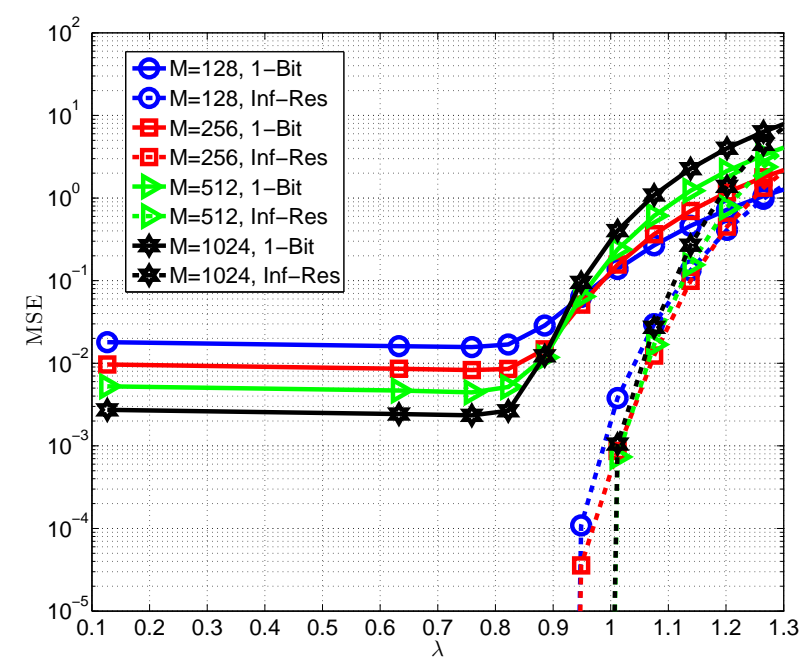

Fig. 6: Average MSE versus $\lambda$ in a 8-user system for different numbers of antennas, $M$, when $P=1$ and $M_{2}=8$.

answer is affirmative under typical system parameter ranges. Fig. 5 suggests that for the transmit power budget $P=1$ the MSE about $10^{-5}$ can be achieved by the proposed algorithm. Since the MSE scales with the power budget, the MSE of the proposed algorithm with the power budget $P$ is about $10^{-5} P$. Using the SER expression in (6) and observing that the MSE is nearly uniform for all symbols before the phase transition, it can be shown that

$$
\mathrm{SER} \approx \bar{g}_{N} Q\left(\frac{c_{1-\text { bit }}^{*}}{2 \sigma(N-1)}-\frac{\sqrt{\mathrm{MSE}}}{\sigma}\right) .
$$

For the typical operating regime of SER (e.g. $10^{-3}-10^{-6}$ ), the contribution of the second term in the $Q$-function can be ignored if it is at least one order of magnitude smaller than the first term, i.e.,

$$
0.1 \frac{c_{1-\text { bit }}^{*}}{2 \sigma(N-1)} \leq \frac{\sqrt{\mathrm{MSE}}}{\sigma} .
$$

For $\mathrm{MSE}=10^{-5} P$ and $c_{1-\text { bit }}^{*}=\sqrt{2 / \pi} \sqrt{2 P}\|\mathbf{h}\|_{2} \approx$ $0.8 \sqrt{2 P M}$, this condition translates to an upper-bound on the number of constellation points as:

$$
N \leq 17.9 \sqrt{M}+1 .
$$

This condition is clearly satisfied in a typical single-user MISO system, with for example 128 antennas and with constellation size at most $N^{2}=2^{12}$. Therefore, the MSE of the proposed one-bit precoding has negligible influence on the SER in such a system.

Next, we show that the constellation range design for the multi-user case proposed in Section VI-A which sets $c_{1 \text {-bit }}^{*}=$ $\sqrt{2 / \pi} \sqrt{\frac{2 P M}{f(K, N)}}$ is also nearly optimal. Toward this aim, we consider a 8-user system with transmit power $P=1$ and 16QAM signaling, i.e., $N=4$. We then vary the constellation

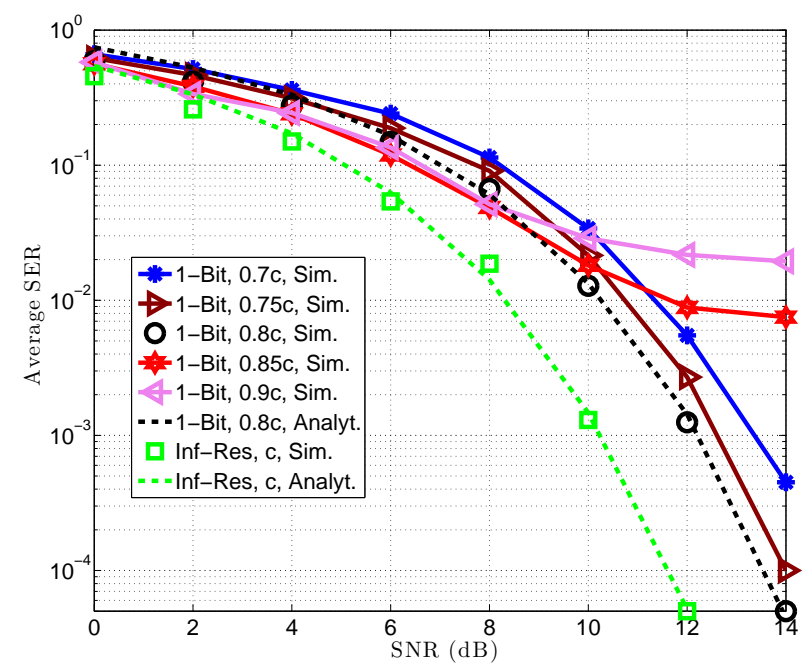

Fig. 7: Average SER versus SNR for different methods in a single-user MISO system with $M=256$ and $N=16$.

range of 16-QAM constellation and plot the average MSE, which for the multi-user setup is defined as

$$
\operatorname{MSE}(\mathbf{s})=\mathbb{E}_{\mathbf{H}}\left\{\left|\sqrt{\frac{P}{M}} \mathbf{H} \mathbf{x}-\mathbf{s}\right|^{2}\right\},
$$

against the parameter $\lambda$ defined as

$$
\lambda=\frac{c}{\sqrt{\frac{2 P M}{f(K, N)}}} .
$$

Fig. 6 shows that for the multi-user scenario, there is a phase transition at $\lambda$ very close to 1 for infinite-resolution ZF precoding, and $\sqrt{2 / \pi} \approx 0.8$ for one-bit precoding, respectively. This verifies our design choices $\sqrt{38}$ and 40 , i.e., $c_{\mathrm{ZF}}^{*}=\sqrt{\frac{2 P M}{f(K, N)}}$ and $c_{1 \text {-bit }}^{*}=\sqrt{2 / \pi} \sqrt{\frac{2 P M}{f(K, N)}}$.

\section{B. SER Analysis for One-Bit Precoding Design}

We now evaluate the SER performance of the proposed one-bit precoding scheme. In the following simulations, the performance of different methods are evaluated using the empirical average SER of the users calculated by averaging the SER over $10^{3}$ channel realizations and in each realization 200 symbols are transmitted, i.e., the coherence time of the channel is 200 symbol transmissions. Further, the signal-tonoise ratio is defined as $\mathrm{SNR}=10 \log _{10}\left(\frac{P}{2 \sigma^{2}}\right)$.

First, we evaluate a single-user communication setup with 256-QAM, i.e., $N=16$. We set the parameter $M_{2}=8$ in the proposed one-bit precoding algorithm. The BS is assumed to be equipped with $M=256$ antennas. In Fig. 7 the performance of the proposed one-bit precoding method with different constellation range designs is compared to the infinite-resolution precoding benchmark with per-symbol power constraint and constellation range of $c=\sqrt{2 P}\|\mathbf{h}\|_{2}$. It can be seen from Fig. 7 that the constellation range of $\sqrt{2 / \pi} c \approx 0.8 c$ achieves the overall best performance, justifying our proposed design (26) for one-bit precoding. 


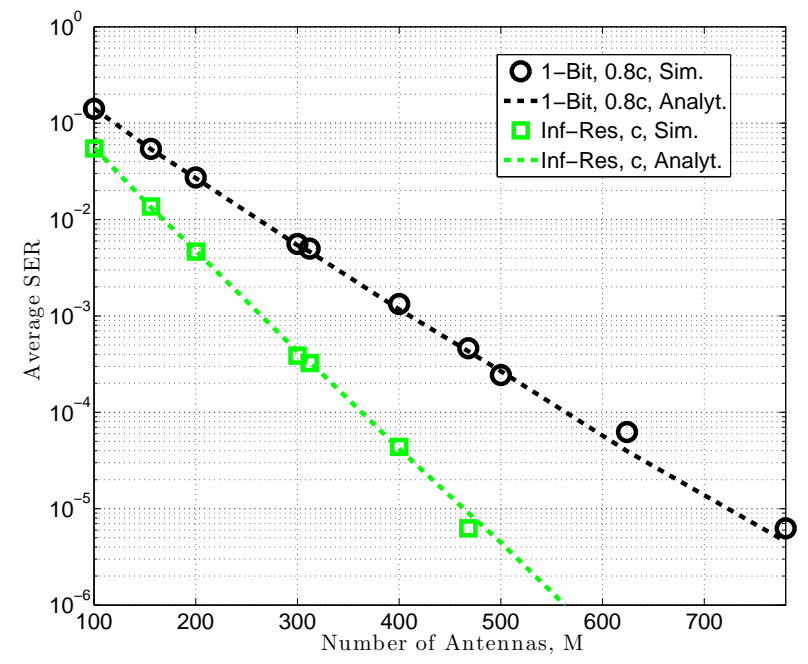

Fig. 8: Average SER versus the number of antennas, $M$, for different methods in a single-user MISO system with $\mathrm{SNR}=-4 \mathrm{~dB}$ and $N=4$.

Fig. 7 also shows that for the one-bit precoding methods with constellation range of larger than $0.8 c$, there is an error floor in the large SNR regime. This is because in the large SNR regime, the average SER is dominated by the worst-case symbols, which are the corner constellation points. One-bit precoding with constellation range larger than $0.8 \mathrm{c}$ leads to high reconstruction MSE for the corner points.

Moreover, Fig. 7 shows that the analytic SER expressions provided in Section $\overline{\mathrm{VI}-\mathrm{C}}$ for the infinite-resolution precoding and the proposed one-bit precoding with constellation range of $0.8 c$ perfectly match the numerical simulation results and the performance gap between the proposed method and the infinite-resolution case is about $2 \mathrm{~dB}$, as predicted by the SER analysis.

Next, we consider a single-user setup in which 16-QAM constellation is employed and the SNR is set to be $-4 \mathrm{~dB}$. For such a system, Fig. 8 plots the average SER against the number of antennas at the BS for the proposed one-bit precoding method as well as the infinite-resolution precoding method. It can be observed from Fig. 8 that the proposed one-bit precoding design can achieve the same performance as the infinite-resolution precoding if the BS in the onebit precoding architecture is equipped with about $50 \%$ more number of antennas as compared to the infinite-resolution case, as predicated by the SER analysis in Section VI-C.

Finally, we consider a multi-user scenario in which $K=$ 8 users are served with a BS equipped with 512 antennas using 16-QAM, i.e., $N=4$. The performance of the proposed one-bit precoding method with $M_{2}=8$ and different design choices for constellation range is evaluated as compared to the infinite-resolution ZF benchmark with constellation range of $c=\sqrt{\frac{2 P M}{f(K, N)}}$ described in Section VI Fig. 9 plots the average SER against the SNR.

It can be observed from Fig. 9 that the analytic SER expression presented in Section VI-C accurately characterizes the average SER in the simulation for the infinite-resolution

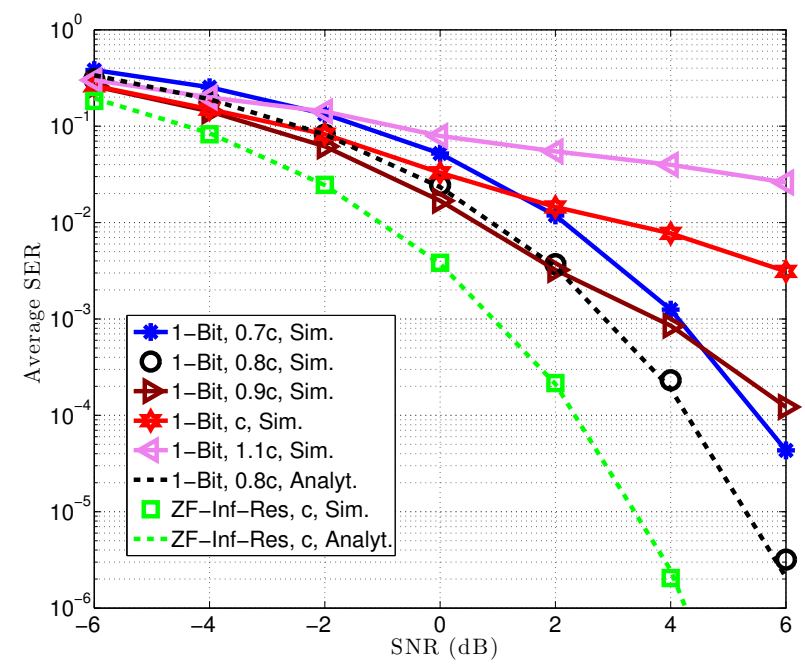

Fig. 9: Average SER versus SNR for different methods in a 8-user MISO system with $M=512$ and $N=4$.

$\mathrm{ZF}$ precoding as well as the proposed one-bit precoding with $\sqrt{2 / \pi} c \approx 0.8 c$. Further, similar to the single-user case, a performance gap of $2 \mathrm{~dB}$ between the proposed method with constellation range $0.8 c$ and conventional $\mathrm{ZF}$ is observed in Fig. 9 Finally, the one-bit precoding schemes with constellation range larger than the proposed design of $0.8 c$ all suffer from an error floor in the high SNR regime, leading to the conclusion that $0.8 c$ is an appropriate design for the constellation range.

\section{CONCLUSION}

This paper considers the one-bit symbol-level precoding architecture for a downlink massive MIMO system. First, we consider the problem of designing the QAM constellation range and the precoder for the single-user scenario in order to minimize the SER. We propose to set the QAM constellation range of one-bit precoding as the optimal constellation range of infinite-resolution precoding reduced by the factor of $\sqrt{2 / \pi}$ or about 0.8 . We then propose a two-step heuristic algorithm to design the precoder, which enjoys a low complexity and exhibits excellent numerical performance. We also generalize the proposed designs for the multi-user scenario. In particular, we first propose constellation range design for the infiniteresolution ZF case then further scale it by $\sqrt{2 / \pi}$ for the one-bit precoding case. Finally, we analytically study the performance of the proposed scheme and show that for largescale antenna arrays, there is a $2 \mathrm{~dB}$ gap between the proposed design and the conventional ZF scheme with per-symbol power constraint. The simulation results verify that the proposed design can achieve a promising performance for large-scale antenna arrays with low-resolution DACs.

\section{REFERENCES}

[1] J. G. Andrews, S. Buzzi, W. Choi, S. V. Hanly, A. Lozano, A. C. K. Soong, and J. C. Zhang, "What will 5G be?" IEEE J. Sel. Areas Commun., vol. 32, no. 6, pp. 1065-1082, June 2014. 
[2] Q. Shi, M. Razaviyayn, Z.-Q. Luo, and C. He, "An iteratively weighted MMSE approach to distributed sum-utility maximization for a MIMO interfering broadcast channel," IEEE Trans. Signal Process., vol. 59, no. 9, pp. 4331-4340, Sept. 2011.

[3] A. Wiesel, Y. C. Eldar, and S. Shamai, "Zero-forcing precoding and generalized inverses," IEEE Trans. Signal Process., vol. 56, no. 9, pp. 4409-4418, Sept. 2008

[4] H. Dahrouj and W. Yu, "Coordinated beamforming for the multicell multi-antenna wireless system," IEEE Trans. Wireless Commun., vol. 9, no. 5, pp. 1748-1759, May 2010.

[5] Y. F. Liu, Y. H. Dai, and Z. Q. Luo, "Max-min fairness linear transceiver design for a multi-user MIMO interference channel," IEEE Trans. Signal Process., vol. 61, no. 9, pp. 2413-2423, May 2013.

[6] X. Zhang, A. F. Molisch, and S.-Y. Kung, "Variable-phase-shift-based RF-baseband codesign for MIMO antenna selection," IEEE Trans. Signal Process., vol. 53, no. 11, pp. 4091-4103, Nov. 2005.

[7] O. El Ayach, S. Rajagopal, S. Abu-Surra, Z. Pi, and R. W. Heath, "Spatially sparse precoding in millimeter wave MIMO systems," IEEE Trans. Wireless Commun., vol. 13, no. 3, Mar. 2014

[8] F. Sohrabi and W. Yu, "Hybrid digital and analog beamforming design for large-scale antenna arrays," IEEE J. Sel. Topics Signal Process., vol. 10, no. 3, pp. 501-513, Apr. 2016

[9] L. Liang, W. Xu, and X. Dong, "Low-complexity hybrid precoding in massive multiuser MIMO systems," IEEE Wireless Commun. Lett., vol. 3, no. 6, pp. 653-656, Dec. 2014

[10] R. H. Walden, "Analog-to-digital converter survey and analysis," IEEE J. Sel. Areas Commun., vol. 17, no. 4, pp. 539-550, Apr. 1999.

[11] C. Svensson, S. Andersson, and P. Bogner, "On the power consumption of analog to digital converters," in Proc. IEEE Norchip Conf., Linköping, Sweden, Nov. 2006, pp. 49-52.

[12] S. K. Mohammed and E. G. Larsson, "Per-antenna constant envelope precoding for large multi-user MIMO systems," IEEE Trans. Commun., vol. 61 , no. 3, pp. 1059-1071, Mar. 2013.

[13] C. Risi, D. Persson, and E. G. Larsson, "Massive MIMO with 1-bit ADC," [Online] Available: http://arxiv.org/abs/1404.7736, Apr. 2014.

[14] J. Mo and R. W. Heath, "Capacity analysis of one-bit quantized MIMO systems with transmitter channel state information," IEEE Trans. Signal Process., vol. 63, no. 20, pp. 5498-5512, Oct. 2015.

[15] Y. Li, C. Tao, G. Seco-Granados, A. Mezghani, A. L. Swindlehurst, and L. Liu, "Channel estimation and performance analysis of one-bit massive MIMO systems," IEEE Trans. Signal Process., vol. 65, no. 15 , pp. 4075-4089, Aug. 2017.

[16] S. Jacobsson, G. Durisi, M. Coldrey, U. Gustavsson, and C. Studer "Throughput analysis of massive MIMO uplink with low-resolution ADCs," IEEE Trans. Wireless Commun., vol. 16, no. 6, pp. 4038-4051, June 2017.

[17] C. Mollén, J. Choi, E. G. Larsson, and R. W. Heath, "Uplink performance of wideband massive MIMO with one-bit ADCs," IEEE Trans. Wireless Commun., vol. 16, no. 1, pp. 87-100, Jan. 2017.

[18] C. Studer and G. Durisi, "Quantized massive MU-MIMO-OFDM uplink," IEEE Trans. Commun., vol. 64, no. 6, pp. 2387-2399, June 2016.

[19] A. Mezghani, R. Ghiat, and J. A. Nossek, "Transmit processing with low resolution D/A-converters," in Proc. IEEE Int. Conf. Electron., Circuits, Syst. (ICECS), Yasmine Hammamet, Tunisia, Dec. 2009, pp. 683-686.

[20] A. K. Saxena, I. Fijalkow, and A. L. Swindlehurst, "On one-bit quantized ZF precoding for the multiuser massive MIMO downlink," in Proc. IEEE Sensor Array Multichannel Signal Process. Workshop (SAM), Rio de Janeiro, Brazil, July 2016, pp. 1-5.

[21] _ "Analysis of one-bit quantized precoding for the multiuser massive MIMO downlink," IEEE Trans. Signal Process., vol. 65, no. 17, pp. 4624-4634, Sept. 2017.

[22] A. Swindlehurst, A. Saxena, A. Mezghani, and I. Fijalkow, "Minimum probability-of-error perturbation precoding for the one-bit massive MIMO downlink," in Proc. IEEE Int. Conf. Acoust., Speech, Signal Process. (ICASSP), New Orleans, LA, USA, Mar. 2017, pp. 6483-6487.

[23] H. Jedda, J. A. Nossek, and A. Mezghani, "Minimum BER precoding in 1-bit massive MIMO systems," in Proc. IEEE Sensor Array Multichannel Signal Process. Workshop (SAM), Rio de Janeiro, Brazil, July 2016.

[24] O. B. Usman, H. Jedda, A. Mezghani, and J. A. Nossek, "MMSE precoder for massive MIMO using 1-bit quantization," in Proc. IEEE Int. Conf. Acoust., Speech, Signal Process. (ICASSP), Shanghai, China, Mar. 2016, pp. 3381-3385.

[25] O. B. Usman, J. A. Nossek, C. A. Hofmann, and A. Knopp, "Joint MMSE precoder and equalizer for massive MIMO using 1-bit quantization," in Proc. IEEE Int. Conf. on Commun. (ICC), Paris, France, May 2017.
[26] L. T. N. Landau and R. C. de Lamare, "Branch-and-bound precoding for multiuser MIMO systems with 1-bit quantization," IEEE Wireless Commun. Lett., vol. 6, no. 6, pp. 770-773, Dec. 2017.

[27] D. B. Amor, H. Jedda, and J. A. Nossek, "16 QAM communication with 1-bit transmitters," in Proc. IEEE Int. ITG Workshop Smart Antennas (WSA), Berlin, Germany, Mar. 2017.

[28] O. Castañeda, T. Goldstein, and C. Studer, "POKEMON: A non-linear beamforming algorithm for 1-bit massive MIMO," in Proc. IEEE Int. Conf. Acoust., Speech, Signal Process. (ICASSP), New Orleans, USA, Mar. 2017, pp. 3464-3468.

[29] S. Jacobsson, G. Durisi, M. Coldrey, T. Goldstein, and C. Studer, "Quantized precoding for massive MU-MIMO," IEEE Trans. Commun., vol. 65 , no. 11 , pp. 4670-4684, Nov. 2017.

[30] _ - "Nonlinear 1-bit precoding for massive MU-MIMO with higherorder modulation," in Proc. IEEE Asilomar Conf. Signals, Syst., Comput., Nov. 2016, pp. 763-767.

[31] B. M. Hochwald, T. L. Marzetta, and V. Tarokh, "Multiple-antenna channel hardening and its implications for rate feedback and scheduling," IEEE Trans. Inf. Theory, vol. 50, no. 9, pp. 1893-1909, Sept. 2004.

[32] H. Jedda, A. Noll, and J. A. Nossek, "PSK precoding in multi-user MISO systems," in Proc. IEEE Int. ITG Workshop Smart Antennas (WSA), Berlin, Germany, Mar. 2017

[33] H. Jedda, A. Mezghani, J. A. Nossek, and A. L. Swindlehurst, "Massive MIMO downlink 1-bit precoding with linear programming for PSK signaling," in Proc. IEEE Workshop Signal Process. Adv. Wireless Commun. (SPAWC), Sapporo, Japan, Jul. 2017.

[34] M. Joham and W. Utschick, "Symbol rate processing for the downlink of DS-CDMA systems," IEEE J. Sel. Areas Commun., vol. 19, no. 1, pp. 61-68, Jan. 2001.

[35] C. Masouros and E. Alsusa, "Dynamic linear precoding for the exploitation of known interference in MIMO broadcast systems," IEEE Trans. Wireless Commun., vol. 8, no. 3, pp. 1396-1404, Mar. 2009.

[36] M. Alodeh, S. Chatzinotas, and B. Ottersten, "Constructive multiuser interference in symbol level precoding for the MISO downlink channel," IEEE Trans. Signal Process., vol. 63, no. 9, pp. 2239-2252, May 2015.

[37] _ - "Symbol-level multiuser MISO precoding for multi-level adaptive modulation," IEEE Trans. Wireless Commun., vol. 16, no. 8, pp. 55115524, Aug 2017.

[38] — , "Energy-efficient symbol-level precoding in multiuser MISO based on relaxed detection region," IEEE Trans. Wireless Commun., vol. 15 , no. 5, pp. 3755-3767, May 2016.

[39] F. Liu, C. Masouros, P. V. Amadori, and H. Sun, "An efficient manifold algorithm for constructive interference based constant envelope precoding," IEEE Signal Process. Lett., vol. 24, no. 10, pp. 1542-1546, Oct. 2017.

[40] C. Masouros and G. Zheng, "Exploiting known interference as green signal power for downlink beamforming optimization," IEEE Trans. Signal Process., vol. 63, no. 14, pp. 3628-3640, July 2015.

[41] J. G. Proakis and M. Salehi, Digital communications, 5th ed. McGrawHill, 2008.

[42] M. Chiani, D. Dardari, and M. K. Simon, "New exponential bounds and approximations for the computation of error probability in fading channels," IEEE Trans. Wireless Commun., vol. 2, no. 4, pp. 840-845, July 2003.

[43] S. Zhang and Y. Huang, "Complex quadratic optimization and semidefinite programming," SIAM Journal on Optimization, vol. 16, no. 3, pp. 871-890, July 2006.

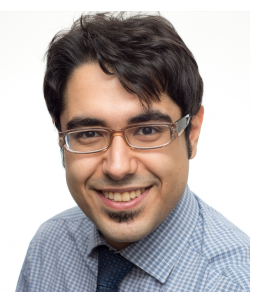

Foad Sohrabi (S'13) received his B.A.Sc. degree in 2011 from University of Tehran, Tehran, Iran, his M.A.Sc. degree in 2013 from McMaster University, Hamilton, ON, Canada, and his Ph.D. degree in 2018 from University of Toronto, Toronto, ON, Canada, all in Electrical and Computer Engineering. During his $\mathrm{PhD}$ study, he was a research intern at BellLabs, Alcatel-Lucent, in Stuttgart, Germany for 6 months. He is currently a Postdoctoral Fellow with University of Toronto, Toronto, ON, Canada. His main research interests include MIMO communications, optimization theory, wireless communications, and signal processing $\mathrm{He}$ received an IEEE Signal Processing Society Best Paper Award in 2017. 


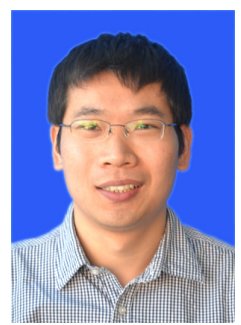

Ya-Feng Liu (M'12) received the B.Sc. degree in applied mathematics in 2007 from Xidian University, Xi' an, China, and the Ph.D. degree in computational mathematics in 2012 from the Chinese Academy of Sciences (CAS), Beijing, China. During his Ph.D. study, he was supported by the Academy of Mathematics and Systems Science (AMSS), CAS, to visit Professor Zhi-Quan (Tom) Luo at the University of Minnesota (Twins Cities) from February 2011 to February 2012. After his graduation, he joined the Institute of Computational Mathematics and Scientific/Engineering Computing, AMSS, CAS, Beijing, China, in July 2012, where he is currently an Assistant Professor. His main research interests are nonlinear optimization and its applications to signal processing, wireless communications, and machine learning. He is especially interested in designing efficient algorithms for optimization problems arising from the above applications.

Dr. Liu has served as a guest editor of the Journal of Global Optimization. $\mathrm{He}$ is a recipient of the Best Paper Award from the IEEE International Conference on Communications (ICC) in 2011 and the Best Student Paper Award from the International Symposium on Modeling and Optimization in Mobile, Ad Hoc and Wireless Networks (WiOpt) in 2015.

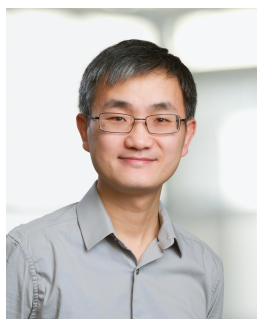

Wei Yu (S'97-M'02-SM'08-F'14) received the B.A.Sc. degree in Computer Engineering and Mathematics from the University of Waterloo, Waterloo, Ontario, Canada in 1997 and M.S. and Ph.D. degrees in Electrical Engineering from Stanford University, Stanford, CA, in 1998 and 2002, respectively. Since 2002, he has been with the Electrical and Computer Engineering Department at the University of Toronto, Toronto, Ontario, Canada, where he is now Professor and holds a Canada Research Chair (Tier 1) in Information Theory and Wireless Communications. His main research interests include information theory, optimization, wireless communications and broadband access networks.

Prof. Wei Yu currently serves on the IEEE Information Theory Society Board of Governors (2015-20). He was an IEEE Communications Society Distinguished Lecturer (2015-16). He is currently an Area Editor for the IEEE Transactions on Wireless Communications (2017-20). He served as an Associate Editor for IEEE Transactions on Information Theory (20102013), as an Editor for IEEE Transactions on Communications (2009-2011), and as an Editor for IEEE Transactions on Wireless Communications (20042007). He is currently the Chair of the Signal Processing for Communications and Networking Technical Committee of the IEEE Signal Processing Society (2017-18) and served as a member in 2008-2013. Prof. Wei Yu received the Steacie Memorial Fellowship in 2015, the IEEE Signal Processing Society Best Paper Award in 2017 and 2008, a Journal of Communications and Networks Best Paper Award in 2017, an IEEE Communications Society Best Tutorial Paper Award in 2015, an IEEE ICC Best Paper Award in 2013, the McCharles Prize for Early Career Research Distinction in 2008, the Early Career Teaching Award from the Faculty of Applied Science and Engineering, University of Toronto in 2007, and an Early Researcher Award from Ontario in 2006. Prof. Wei Yu is a Fellow of the Canadian Academy of Engineering, and a member of the College of New Scholars, Artists and Scientists of the Royal Society of Canada. He is recognized as a Highly Cited Researcher. 